\title{
Accommodation and stereoacuity
}

\author{
S. M. LLRIA and JO ANN S. KINNEY \\ Varal Submarine Vedical Center. Groton. Connecticut (10.311)
}

\begin{abstract}
Stereoacuity and resolution acuity were measured 1 li through apertures set at various distances from 0 and 12) through plus lenses producing an accommodative error for the target equal to that produced by the presence of the apertures. Stereoacuity was degraded by the apertures but not by the lenses. whereas resolution acuity was degraded by the lenses but not by the apertures. Although stereoacuity progressively declined with decreasing target distance. it did not change significantly if accommodation remained constant. The decline of stereoacuity in the water is attributed to increased accommodation resulting from different sources and to a "Ganzfeld" effect of the typical underwater scene.
\end{abstract}

Stereoacuity under water is affected by the presence of peripheral stimuli (Luria \& Kinney. 1972). Those which are near the plane of the $O$ degrade stereoacuity. whereas stimuli near the plane of the target improve it. compared to acuity in the absence of peripheral stimuli.

A recent report by Hennessy and Leibowitz (1971) seemed to provide an explanation. They found that accommodation for a target is affected when the target is viewed through an aperture. The eve tries to accommodate for both target and aperture; to the extent that accommodation for the aperture occurs, accommodation for the target is in error. As the aperture is moved closer to the eye, the error of accommodation for the target increases. It seemed likely, therefore, that in our experiments the decline in stereoacuity as peripheral stimuli were moved closer to the $O$ was due to a compromise in accommodation. for them and the target-the same compromise in accommodation found by Hennessy and Leibowitz. Furthermore, since the facemask itself acts as an aperture very close to the diver, it might also produce errors of accmmodation which would contribute to the marked deterioration of stereoacuity in the water.

To show that this is, in fact. the explanation, it is necessary (1) to measure acuity under conditions similar to those which Hennessy and Leibowitz found to affect accommodation. and (2) to show that errors in accommodation of the magnitude found by them produce changes in stereoacuity similar to those produced in our experiments in the water. Accommodation could affect stereoacuity in at least two different ways. An error in accommodation for the target (resulting from the aperture) could degrade stereoacuity by producing a blurred image, or merely the activation of the accommodation mechanism might affect stereoacuity.

To test these alternatives, plus lenses of the appropriate power were used to blur the retinal image during measurements of both resolution and stereoacuity. To test the effect of activation of the accommodative mechanism. minus lenses-for which Os can accommodate-were introduced in amounts equivalent to the degree of accommodation produced by the presence of Hennessy and Leibowitz's apertures.

\section{ACUITY THROUGH AN APERTURE}

\section{Apparatus and Procedure}

The experimental situation resembled that described by Hennessy and Leibowitz (1971). The $O$ sat in the center of a large room $6 \mathrm{~m}$ from a white wall against which a series of Landolt $C s$ or a three-rod Howard-Dolman apparatus could be placed. Both the wall and the white backplate of the Howard-Dolman were illuminated to $5 \mathrm{fl}$. At the viewing distance of $6 \mathrm{~m}$. the Howard-Dolman subtended $1.25 \times 3.5 \mathrm{deg}$ of visual angle.

A movable screen. covered with white bainbridge board and measuring $1.7 \mathrm{~m}$ high $\mathrm{x} 2.5 \mathrm{~m}$ wide. could be positioned from $4 \mathrm{~m}$ to $7.5 \mathrm{~cm}$ from $O$ at a constant luminance of $16 \mathrm{fl}$. At distances of $7.5 \mathrm{~cm}$ and $0.5 \mathrm{~m}$, the screen filled $O$ 's field of view. of course; at $1 \mathrm{~m}$. it subtended $60 \times 70 \mathrm{deg}$ of visual angle. and at $4 \mathrm{~m}$. it subtended $23 \times 32 \mathrm{deg}$. It thus constituted a surround which. however, varied in size.

The center of the screen held one of a series of replaceable apertures. With the screen set at 4,1 , or $0.5 \mathrm{~m}$ from 0 , the apertures permitted a rectangular view of the target area corresponding to the size of the Howard-Dolman apparatus. $1.25 \times 3.5 \mathrm{deg}$. At 4 and $1 \mathrm{~m}$, only one rectangular aperture was used: at 0.5 and $7.5 \mathrm{~cm}$. two apertures were necessary; one adjustable for interpupillary separation. With the screen $7.5 \mathrm{~cm}$ away, apertures smaller than the diameter of the pupil were necessary to restrict the field of view to the size of the Howard-Dolman. Since this resulted in the appearance of a film before O's eyes. the aperture size was increased until the film disappeared. The resulting apertures were $7 \mathrm{~mm}$ in diam and permitted a circular field of view of slightly more than 5 deg.

The $O$ sat with his head positioned by a chin- and foreheadrest. He was carefully instnucted as to the importance of positioning the adjustable apertures correctly, holding his head motionless. and continually checking to insure that both eyes had an unobstructed view of the target.

The four positions of the screen were presented in counterbalanced order. The stereoacuity thresholds were alway's measured first, followed by the resolution thresholds. Each set of thresholds was taken in a different counterbalanced order for each $O$. Viewing time was not limited: $O$ was allowed to look until he had reached a decision.

Both resolution and stereoacuity thresholds were measured by the method of constant stimuli. For the former. a set of four to eight Landolt Cs was chosen which encompassed O's acuity. and 


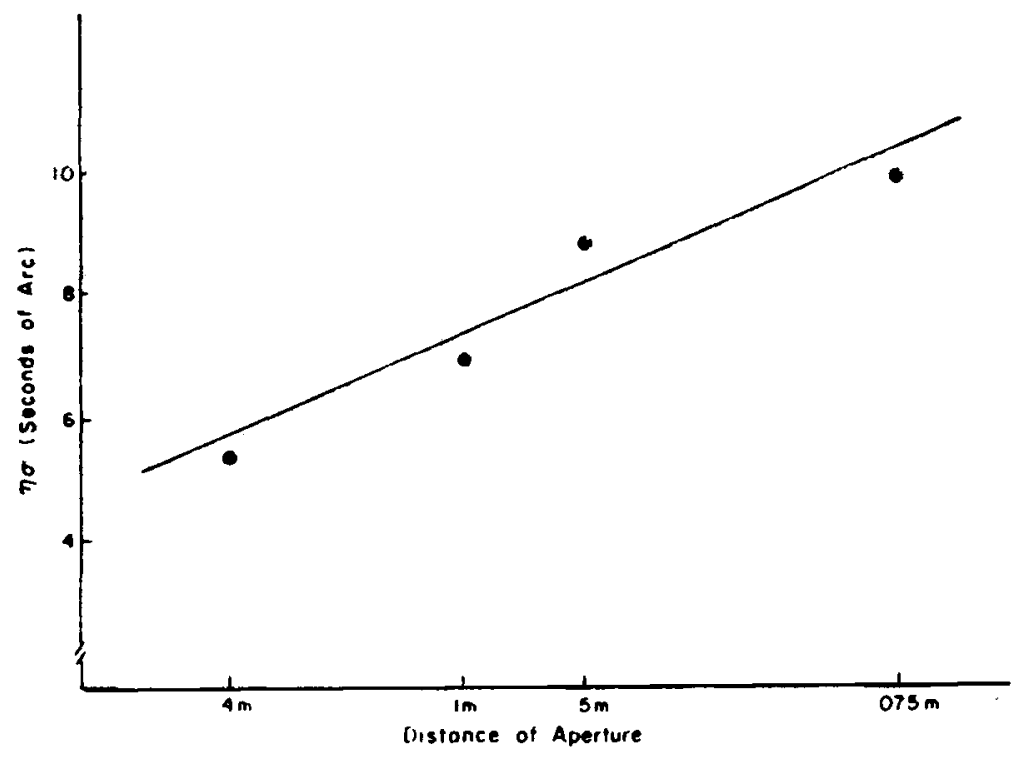

Fig. 1. Mean precision of stereoacuity (seconds of arc) for a target at $6 \mathrm{~m}$ seen through an aperture set at various distances from 0 .

each size tarect was presented in random order. The $C$ was prewented "ith the gap in one of four positions, 3.6, 9. or 12 o'clock. The'se positions were given in haphazard order, but care was taken to present the 3 and $90^{\circ}$ clock positions half the time

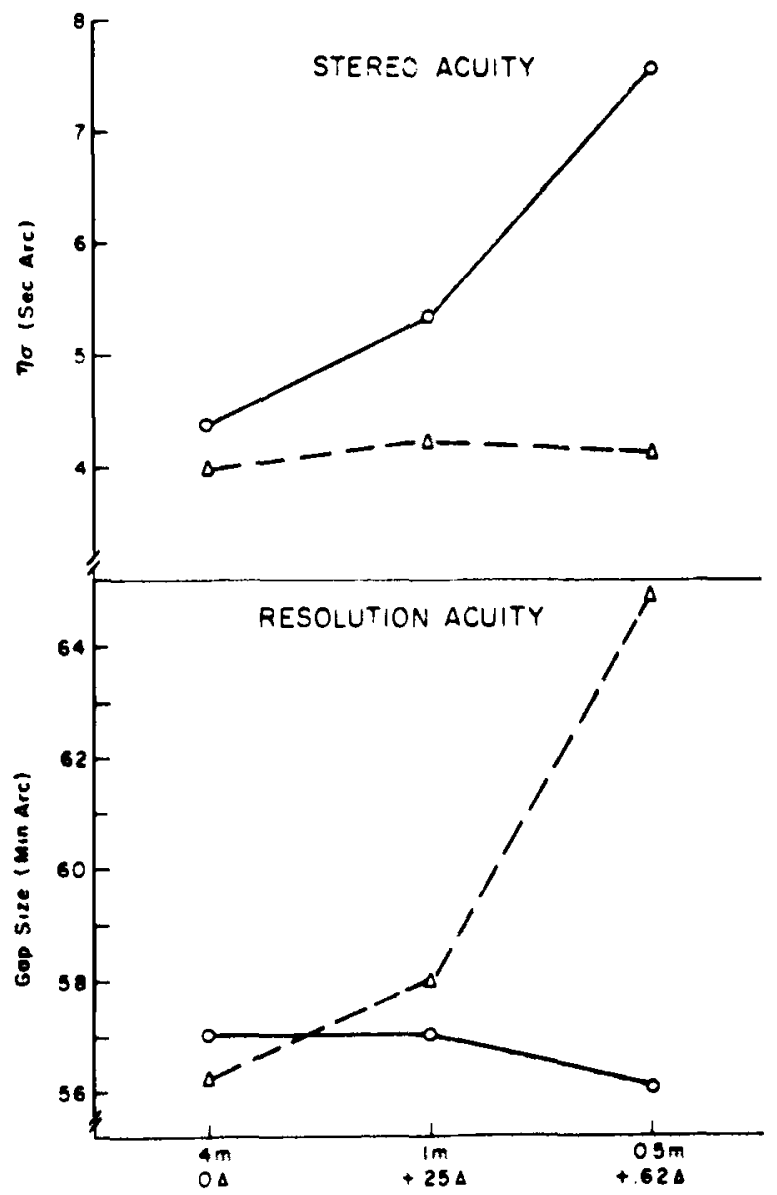

Fig. 2. Mean scsolution acuity and precision of stereoacuity for targets at $6 \mathrm{~m}$ seen either through an aperture at various distances (solid line) from $\mathrm{O}$ or through plus lenses (broken line). and the 6 and 12 o'clock positions half the time. A frequency of seeing curve was drawn on cumulative probability paper, and the $50 \%$ size was taken as the threshold.

The stereoacuity data are given in terms of variability of the equidistance setting. The middle (movable) rod was set at various positions. and O judged either "closer" or "farther" than the two outside rods. Again, a frequency of seeing curve was drawn on cumulative probability paper and the standard deviations read directly from the graph.

\section{Observers}

Staff members of the laboratory and enlisted men from the Naval Submarine School served as Os. All enjoyed at least 20/25 uncorrected acuity in each eye, according to the Bausch and Lomb Ortho-Rater.

\section{Results}

The mean stereoacuity thresholds for 12 Os for the four distances of the aperture are plotted in Fig. 1. Mean stereoacuity progressively declined as the aperture was moved closer to $\mathrm{O}\left(\mathrm{\chi r}^{2}(3)=16.1, \mathrm{p}<.001\right.$, Friedman ANOVA by ranks). Resolution acuity remainen unchanged $\left(x \mathrm{r}^{2}(3)=1.4, \mathrm{p}<.75\right)$.

\section{ACUITY THROUGH POSITIVE LENSES}

The next step was to see if the change in stereoacuity could be produced by changes in accommodation of the magnitude which were induced by the presence of the aperture. According to Hennessy and Leibowitz, the aperture at $1 \mathrm{~m}$ from $\mathrm{O}$ induced a mean error of accommodation of about 0.25 diopter, and the aperture at $0.5 \mathrm{~m}$ induced an error of about 0.6 diopter. Both stereoacuity and resolution acuity for targets at $6 \mathrm{~m}$ were the refore tested with $\mathrm{O}$ wearing $0.25-\mathrm{D}$ and $0.62 \cdot \mathrm{D}$ lenses and also empty trial frames. in counterbalanced order. The results for 12 Os are plotted in Fig. 2.

It is clear that the screen and lenses have opposite 
effects. Mean stereoacuity declined as the screen was moved closer to $O\left(Y r^{2}(2)=5.46 . p<.10\right)$ but was not systematically affected $\left(\mathrm{Xr}^{2}(2)=0.79 . \mathrm{p}<.70\right)$ by the positive lenses which produce the refractive error of the magnitude which Hennessy and Leibowitz found was produced by the screen. Conversely. resolution acuity was significantly degraded by the lenses $\left(\mathrm{Xr}^{2}(2)=7.17\right.$. $\mathrm{p}<.05)$. but it was not degraded by the screen $\left(\chi \mathrm{r}^{2}(2)=\right.$ 2.54. $p<.25)$. It is thus evident that the changes of accommodation induced by the presence of the screen are enough to degrade resolution acuity but not stereoacuity. The decline of stereoacuity in the presence of the screen is. therefore. not attributable to errors of accommodation.

\section{STEREOACUITY UNDER "EQUIVALENT" ACCOMMODATION}

Another factor which affects stereoacuity is degree of accommodation. or accommodative effort (Luria \& Kinney, 1972). Our previous study showed that stereoacuity was degraded by the presence of a -2.0-D spherical lens even though the Os reported that the target apparatus was in focus. We next sought to find out if equal accommodative efforts resulted in equal stereoacuity. When the eye is accommodated for a target at $2 \mathrm{~m}$, it is exerting an accommodative force of 0.5 diopter. If a -0.25 -D lens is introduced into the latter situation, $O$ must now exert a force of accommodation of 0.50 diopter to focus on the target at $4 \mathrm{~m}$ while overcoming the effect of the lens. Thus. his magnitude of accommodation is now the same as for the target at $2 \mathrm{~m}$.

Stereoacuity thresholds were next measured for $21 \mathrm{Os}$ under three conditions, presented in counterbalanced order: with the target at $7 \mathrm{~m}$ (accommodation = 0.14 diopter) and at $1.12 \mathrm{~m}$ (accommodation = 0.89 diopter), and with a $-0.75 \cdot \mathrm{D}$ lens at the $7 \cdot \mathrm{m}$ distance, which equated the degree of accommodation at that viewing distance to the degree of accommodation at $1.12 \mathrm{~m}$. A second Howard-Dolman apparatus was constructed for use at $1.12 \mathrm{~m}$, which duplicated the visual subtense of the components at $7 \mathrm{~m}$. The results are given in Table 1. The introduction of the $-0.75-\mathrm{D}$ lens significantly degraded stereoacuity $(p>.005$, Wilcoxon, one-tailed), as did the reduction of the target distance to $1.12 \mathrm{~m}$ ( $p>.005$. Wilcoxon, one-tailed). However, there was no statistically significant difference between the thresholds at $1.12 \mathrm{~m}$ and $7 \mathrm{~m}$ when the lenses were worn ( $p>80$. Wilcoxon, two-tailed). These results indicate that equal effort of accommodation results in equal acuity thresholds. This conforms to previous findings that thresholds for targets at different distances are equal when the important variables are held constant (Ogle. 1958; Jameson \& Hurvich. 1959).
Table 1

Precision of Stereoacuity ( $r_{\sigma}$ in Seconds of Arc) Linder Equivalent Accommodation at $T$ wo $T$ arget Distances Compared With a Third Distance

\begin{tabular}{|c|c|c|c|}
\hline \multirow[b]{2}{*}{$S$} & \multicolumn{3}{|c|}{ Target Distance and Power of Lens } \\
\hline & $7 \mathrm{~m}$ & $\begin{array}{l}7 \text { m with } \\
1-.75 د 1\end{array}$ & $1.12 \mathrm{~m}$ \\
\hline SL. & 1.24 & 1.62 & 1.57 \\
\hline C.II & 1.01 & 3.05 & 2.09 \\
\hline EX & 1.74 & 1.74 & 1.57 \\
\hline EG & 3.56 & 6.12 & 2.92 \\
\hline$A X$ & 3.74 & 2.50 & 6.26 \\
\hline BT & 2.85 & 1.74 & 3.65 \\
\hline EH & 3.39 & 3.26 & 2.92 \\
\hline IIS & 1.25 & 1.50 & 1.67 \\
\hline EY & 1.70 & 2.12 & 3.65 \\
\hline BN & 0.67 & 1.55 & 3.65 \\
\hline $\mathrm{HX}$ & 2.65 & 2.15 & 3.13 \\
\hline $11 x$ & 0.67 & 1.24 & 1.36 \\
\hline $\mathrm{BH}$ & 23.71 & * & 9.88 \\
\hline $\mathrm{CS}$ & 1.10 & 2.85 & +82 \\
\hline DMI & 3.05 & 3.73 & 5.00 \\
\hline GS & $1.0 ?$ & 2.91 & 2.00 \\
\hline GI & 1.37 & 5.28 & 4.40 \\
\hline $\mathrm{HX}$ & 2.59 & 1.76 & 3.12 \\
\hline$M Y$ & 4.05 & $*$ & 12.50 \\
\hline$\therefore L$ & 6.99 & 8.48 & 6.75 \\
\hline RE & 1.50 & 2.38 & 2.82 \\
\hline Mean & 3.33 & 4.39 & 4.08 \\
\hline Median & 1.74 & 2.50 & 3.13 \\
\hline
\end{tabular}

*Could not be measured owing to limitations of the apparatus.

\section{STEREOACUITY AND APPARENT TARGET DISTANCE UNDER WATER}

The results in the preceding section made it quite clear that if critical variables such as accommodation are not held constant, then target distance exerts a major effect on stereoacuity. This is of major interest for the study of stereoacuity under water, of course. since the optical distance of submerged objects is three-quarters their actual distance. Now, it has long been reported that stereoacuity declines with decreasing target distance, but previous studies have naturally kept constant such stimulus variables as the subtense of the test rods and their separation (Graham, 1965). When the optical distance of a target is altered in the water. these variables do not, of course, remain constant. It therefore seemed worthwhile repeating such an experiment without the usual controls. We accordingly measured stereoacuity at two pairs of distances, 6 and $4.5 \mathrm{~m}$, also 2.5 and $1.9 \mathrm{~m}$, in order to see the effect of reducing a target distance by the amount that would appear to take place in the water. These four conditions were given in counterbalanced order. The results for eight Os are shown in Fig. 3.

Mean precision of stereoacuity declined significantly with decreasing distance $\left(X \mathrm{I}^{2}(3)=15.5 . \mathrm{p}<.01\right)$. Thus, the refractive distortion which produced a virtual image 


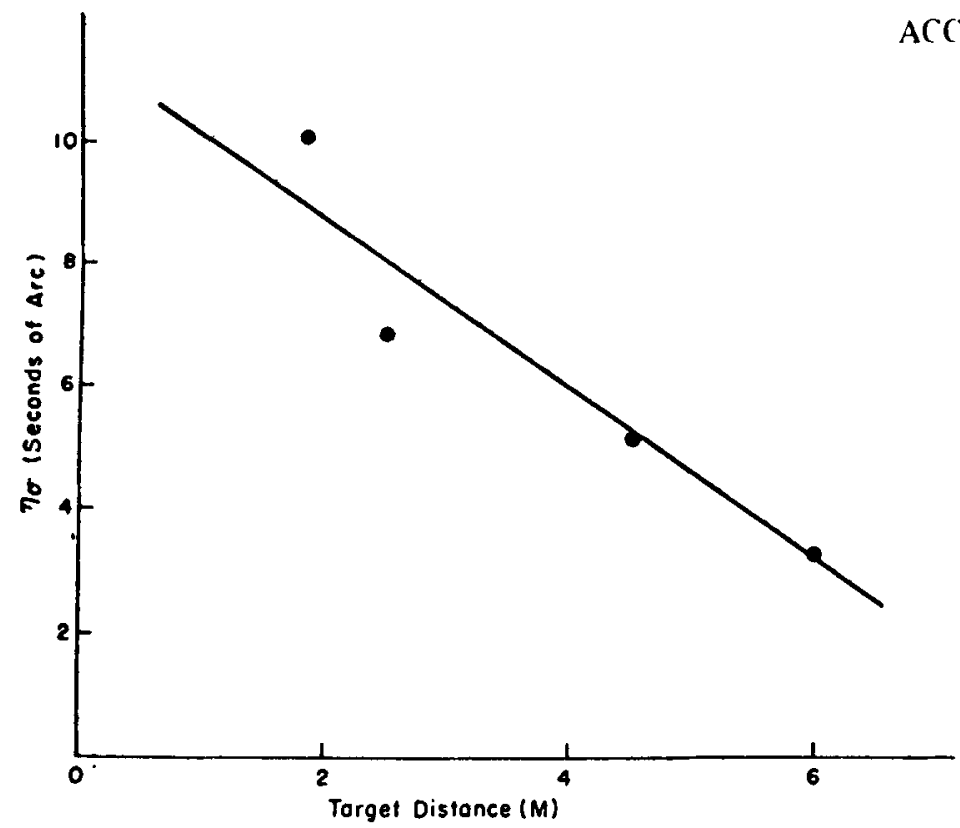

Fig. 3. Mean precision of stereoacuity for a target set at various distances from 0 .

of an underwater target at three-quarters its true distance is enough to produce an appreciable degradation of stereoacuity.

\section{DISCUSSION}

Stereoacuity is affected by the presence of an intervening aperture. As the aperture is moved closer to $\mathrm{O}$, stereoacuity is progressively degraded. Resolution acuity, on the other hand, is unaffected. Since Hennessy and Leibowitz have shown that the intervening aperture affects accommodation, it is tempting to conclude that the induced errors of accommodation are large enough to disturb stereoacuity but too small to affect resolution acuity.

Coupled with the assumption that there are fluctuations of accommodation and that under unlimited exposure durations there eventually occurs at least a brief moment of correct accommodation, such an explanation would conform to the fact that only a very short exposure time, about $0.1 \mathrm{sec}$, is needed to establish maximum resolution acuity (Riggs, 1965) but that maximum stereoacuity is found "when the duration is three seconds and longer, while for shorter durations down to 0.2 second, there is a fourfold to fivefold decrease in acuity [Ogle, 1950]."

The second experiment, however, shows that errors of accommodation of the magnitude induced by the aperture are not the explanation. The prevention of correct accommodation with plus spherical lenses does not greatly affect stereoacuity but does degrade resolution acuity. We are, therefore, led to the conclusion that it is not the errors of accommodation that degrade stereoacuity but rather the magnitude of accommodation (and convergence) that is required to focus the target that affects stereoacuity. This is indicated by the fact that the introduction of negative spherical lenses of rather low power, whose effects $O$ can overcome with his own accommodation, leads to a reduction of stereoacuity. It is also indicated by the reduction in stereoacuity with decreasing target distance, although once again the target is completely in focus. The degradation of stereoacuity produced by the introduction of peripheral stimuli near $\mathrm{O}$ appears to be the result not of retinal blur from an induced error of accommodation for the target, but is rather the result simply of increased accommodation induced by the presence of the additional stimuli.

In summary, we conclude that two factors cause the degradation of stereoacuity through the apertures and in the water (Ross, 1967; Luria \& Kinney, 1968). One is the Ganzfeld effect (Luria, 1969, 1971) which is typical of the underwater scene. The other is the degree of accommodation. Increased accommodation results primarily from the reduced apparent distance of the target in the water. Also contributing to it is the presence of other objects in the field of view which are closer than the target. The eye attempts to accommodate for them as well as for the primary target. and there is thus a greater magnitude of accommodation than is required. This phenomenon is more powerful in the water because of the profound decrease in the contrast of targets with increasing distance. Thus, nearby objects are more visible and exert a disproportionate effect on accommodation compared to their effect in air.

\section{REFERENCES}

Graham, C. H. Visual space perception. In C. H. Graham (Ed.). Vision and visual perception. New York: Wiley, 1965. P. 526. Hennessy, R. T.. \& Leibowitz. H. W. The effect of a peripheral 
stumulus on accommodation. Perception \& Psychophysics. 19?1. 10. 129-132.

lameson. D.. \& Hurich. L. M. Note on factors influencing the relution between stereoscopic acuity and observation distance. Journal of the Optical Society of America. 1959.49.639.

Luria. S. M. Stereoscopic and resolution acuity with various fields of view. Science. 1969, 164. 452-453.

Luria. S. M. Effect of limited peripheral cues on stereoacuity. Psychonomic Science. 1971. 24. 195-196.

Luria. S. M., \& Kinney. J. A. S. Stereoscopic acuity under water. American Journal of Psychology. 1968. 81. 359-366.

Luria. S. If.. \& Kinney. J. A. S. Peripheral stimuli and - stereoacuity under water. Perception \& Psychophysics. 1972. 11. $437-440$.
Ogle. K. $\therefore$. Researches in binocular rision. Philadelphia: Saunders. 1950. P. 136

Ogle, $K$. $\therefore$. Note on stereoscopic acuity and observation distance. Journal of the Optical Society of Ameriea. 1958. 48. 794-798.

Riggs, L. A. Visual acuity. In C. H. Graham (Ed.). Vision and visual perception. New York: Wiley, 1965. P. 339

Ross. H. E. Stereoscopic acuity under Water. In J. X. Lythgoe and J. D. Woods (Eds.). inderwater association reports. 1966-67. London: T. G. Williams Industrial \& Research Promotions. Ltd.. 1967. Pp. 61-64.

(Received for publication June 30, 1972: revision received September 13.1972.) 